\title{
Spatial-dynamics of Hypoxia and Fisheries: \\ The Case of Gulf of Mexico Brown Shrimp
}

\author{
Martin D. Smith, ${ }^{1, *}$ Frank Asche, ${ }^{2}$ Lori S. Bennear, ${ }^{3}$ Atle Oglend ${ }^{4}$
}

1. Dan and Bunny Gabel Associate Professor of Environmental Economics, Nicholas School of the Environment and Department of Economics, Duke University, Box 90328, Durham, NC 27701, Email: marsmith@ duke.edu, ph: (919) 613-8028, fax: (919) 684-8071

2. Professor, Department of Industrial Economics, University of Stavanger, 4036 Stavanger, Norway, Email: frank.asche@uis.no

3. Associate Professor of Environmental Economics and Policy, Nicholas School of the Environment, Department of Economics, and Sanford School of Public Policy, Duke University, Box 90328, Durham, NC 27701, Email: lori.bennear@duke.edu

4. Associate Professor, Department of Industrial Economics, University of Stavanger, 4036

Stavanger, Norway, Email: atle.oglend@uis.no

* Corresponding author.

Acknowledgments: Financial support for this research was provided by the National Oceanic and Atmospheric Administration Grant\# NA09NOS4780235 and by the Research Council of Norway. The authors thank Jim Nance and James Primrose for providing the U.S. shrimp data; Kevin Craig for providing hypoxia data; Justin Kirkpatrick for valuable research assistance; and Josh Abbott, Chris Timmins, Eli Fenichel, Steve Polasky, Jim Sanchirico, conference participants at AERE 2012 and NAAFE 2013; seminar participants at Yale University, Appalachian State University, and the University of Wisconsin; two anonymous referees; and the Guest Editor, Sherry Larkin, for helpful comments. 


\begin{abstract}
We analyze the Gulf of Mexico brown shrimp fishery and the potential impacts of a large seasonal area of hypoxia (low dissolved oxygen) that coincides with the peak shrimp season. A spatial-dynamic bioeconomic simulation embeds three biological impacts on shrimp: mortality, growth, and aggregation on hypoxic edges. Hypoxia creates feedbacks in the bioeconomic system, altering catch and effort patterns. System changes propagate over space to affect areas that do not experience hypoxia. Areas that might otherwise be considered controls in a natural experiments framework are contaminated by the ecological disturbance through spatial sorting. Aggregate predictions from simulations are similar to empirical fishery data. Average shrimp size and total landings are negatively correlated, as are hypoxic severity and landings. Shrimp size and hypoxic severity are only weakly negatively correlated. Growth overfishing, which varies with recruitment success and with ecological disturbances, is a key mediating effect.
\end{abstract}

\title{
JEL Code Q22.
}

Key words: Shrimp, hypoxia, spatial-dynamics, dead zone, bioeconomics, growth overfishing 


\section{Introduction}

There are numerous ecological shocks that can threaten renewable resource extraction and flows of non-extractive ecosystem services. Examples in the marine environment include temperature variation due to phenomena as varied as El Niños and climate change, ocean acidification, the proliferation of large dead zones in coastal waters triggered by nutrient pollution, and technological disasters, such as the Deepwater Horizon oil spill. Natural scientists can readily measure indicators of these problems by tracking ocean temperature, $\mathrm{pH}$, bottom dissolved oxygen, and the presence of hydrocarbons in seawater, respectively. But disentangling whether an ecological disturbance is economically consequential is much more onerous.

In this article, we analyze the Gulf of Mexico brown shrimp (Farfantepenaeus aztecus) fishery and potential impacts of a large seasonal area of hypoxia (low dissolved oxygen)— known popularly as the Dead Zone — that coincides with the height of the shrimp season (Rabalais et al. 2002). The Gulf shrimp fishery is large (typically over 100,000 metric tons of landings per year), and the spatial extent of hypoxia is substantial (up to 22,000 square kilometers), yet natural scientists have had difficulty linking large-scale seasonal hypoxia to fishery losses (Diaz and Rosenberg 2008). There is correlation between hypoxic severity and aggregate shrimp catch in the Gulf (Zimmerman and Nance 2001), but there are no causal inference studies of the phenomenon. Economists have had some success detecting effects of hypoxia on fisheries using micro data in other settings (Massey, Newbold, and Genter 2006; Huang, Smith, and Craig 2010; Huang et al., 2012), and potential effects have been explored with empirically grounded bioeconomic simulations (Knowler, Barbier, and Strand 2001; Smith 2007). Empirical work with micro data has focused on hypoxia in estuaries along the east coast of the United States and effects on catch rates. In North Carolina, for instance, brown shrimp are exposed to low dissolved oxygen as juveniles but are subsequently caught 
as adults in oxygenated waters; the lagged effect of hypoxia translates into a $13 \%$ decline in catch (Huang, Smith, and Craig, 2010).

In contrast to hypoxia in estuaries along the East Coast, the Gulf of Mexico hypoxic area spans a much larger area, occurs in open water, potentially affects a much larger fishery (the Gulf shrimp fishery is between one to two orders of magnitude larger than the North Carolina fishery), and coincides with the adult rather than juvenile life stage of shrimp. Despite the prominence of the Gulf hypoxic area, we also have less temporally resolved environmental data than in, for instance, the case of the Neuse River of North Carolina. As such, we have limited empirical information on the effects of hypoxia on Gulf fisheries and little understanding, even theoretically, about the potential bioeconomic outcomes. While lab experiments demonstrate that hypoxia slows down shrimp growth and delays molting, and mortality is a limiting case if hypoxia is severe enough, there is a countervailing force at play to limit the effect in the fishery. In particular, marine ecologists have shown that shrimp tend to congregate on the edges of hypoxic areas, making them easier to catch (Craig and Crowder 2005; Craig 2012). These forces raise questions about the net effect of hypoxia on the shrimp fishery. How would these effects unfold over time and space? How can empirical researchers detect net effects in landings data given the high degree of inter-annual recruitment variability present in the shrimp fishery?

In environmental economics, causal inference studies of non-random interventions often use spatial characteristics as sources of identification. Examples include effects of regulation on air quality (Auffhammer, Bento, and Lowe 2009), roads on deforestation (Deng et al. 2011), terrestrial protected areas on deforestation (Andam et al. 2008), and marine reserve on fisheries catches (Smith, Zhang, and Coleman 2006). There may be similar opportunities to study the impacts of Gulf hypoxia using its spatial and temporal variation. However, causal approaches to observational data all require separation between treatment and control groups 
such that the treated group does not influence the control group. The simulations we conduct below raise questions about this separation. Before pursuing a particular treatment effects approach, we first must understand how a spatial-dynamic bioeconomic system would affect the data-generating process and the extent to which a hypoxic signal would be transmitted in observational data.

In this article, we conduct a series of spatial-dynamic bioeconomic simulations and compare results to aggregate empirical data on the Gulf shrimp fishery. The simulations allow us to understand the ways in which known connections between hypoxia and biological impacts translate into measurable outcomes in fishery data. The simulations also produce predictions about aggregate-level data that can be compared to empirical aggregate data and shed light on why scientists have had difficulty attributing fishery losses to hypoxia. We find that hypoxia can interact with growth overfishing — an existing market failure (Caillouet, Hart, and Nance 2008) - by drawing latent effort into the fishery, affecting spatial sorting of the fleet, and altering the size distribution of caught shrimp. The countervailing biological channels through which hypoxia affects shrimp can lead to "treatment effects" of hypoxia that are nonlinear and can even be non-monotonic. Moreover, the spatial-dynamic nature of the problem can contaminate potential statistical controls that might otherwise be used for causal inference. The simulations and aggregate-level empirical analysis imply that alternative empirical strategies will be required to analyze the impacts of hypoxia that can accommodate these nonlinearities and spatial dynamics.

\section{A Spatial-dynamic Bioeconomic Simulation Model}

To understand the potential effects of hypoxia on an annual species like shrimp, we conduct a series of numerical experiments using a spatial-dynamic bioeconomic model. The experiments allow us to explore the three channels through which hypoxia may affect 
biological outcomes, which, in turn, affect economic outcomes: 1) hypoxia adds to baseline natural mortality (hereafter mortality effect), 2) hypoxia increases catchability as organisms aggregate along the edges of hypoxic zones (hereafter catchability effect), and 3) hypoxia slows growth (hereafter growth effect). The mortality effect is a tautology of sorts; when water becomes sufficiently low in oxygen, organisms die if they are unable to move to oxygenated areas. Whether there are sufficiently large hypoxic (or anoxic) areas within the spatio-temporally varying Gulf of Mexico Dead Zone to affect shrimp mortality is an open question. The catchability effect follows empirical work showing aggregation of shrimp, other fished species, and fishing vessels along edges of the hypoxic zone in Gulf of Mexico (Craig and Crowder 2005; Craig 2012). The growth effect is consistent with experiments that show slowed lipid uptake and delayed molting in shrimp that are exposed to low oxygen (Craig 2012). Although we abstract away from shrimp migratory behavior in our model, shrimp migrations may further complicate the ability to disentangle countervailing effects of hypoxia on the fishery in observational studies.

\section{Numerical Model and Parameterization}

The biological model is a single-cohort, size-structured model as in Huang and Smith (2011). We assume exogenous recruitment in each location with deterministic and stochastic components. ${ }^{1}$ There are nine locations (zones), and for simplicity we assume that shrimp do not move across zones. Recruitment in each year $(y)$ in each zone $(j)$ is given by:

$$
N_{0, j, y}=\widetilde{N}\left(1+\varepsilon_{j, y}\right) \theta_{j}
$$

where $\widetilde{N}$ is baseline recruitment, $\varepsilon_{j, y}$ is a uniform random variable defined on the unit interval, and $\theta_{j}$ allocates recruits across space. The individual shrimp are subject to natural and fishing mortality such that:

$$
N_{t, j, y}=N_{0, j, y} e^{\sum_{s}-m_{s}+\sum_{s}-f_{s}},
$$


where $t$ indexes time within the year, $s$ indexes time steps for periods 0 to $t$ - 1 within the year, $m$ is natural mortality, and $f$ is fishing mortality. We suppress the $y$ and $j$ subscripts while developing the model until we turn to modeling hypoxia, recognizing that all endogenous variables are year-specific and hypoxia will vary across zones. Individuals grow to length, $L$, according to von Bertallanfy growth with terminal length $L_{\infty}$ and slope parameter $\delta$ :

$$
L_{t}=L_{\infty}\left(1-e^{-\delta t}\right) \text {. }
$$

Natural mortality decreases in size (Minello, Zimmerman, and Martinez 1989). One

explanation is that fewer predators have large enough jaws to eat larger individuals:

$$
m_{t}=\beta\left(L_{t}\right)^{\mu}
$$

where $\beta>0$ and $\mu<0$. The implication is that instantaneous natural mortality is decreasing in age. Fishing mortality is the product of total effort for the fleet, $E$, and catchability, $q$ :

$$
f_{t}=q E_{t}
$$

We return to fishing mortality in the economic model in which we endogenize fishing effort. Biomass is based on an allometric conversion from fish length to weight:

$$
w_{t}=\omega\left(L_{t}\right)^{\gamma},
$$

where $\omega>0$ and $\gamma<0$. Instantaneous harvest, $H_{t}$, in a location is a function of individuals, natural mortality, fishing mortality, and shrimp weight:

$$
H_{t}=\frac{f_{t}}{f_{t}+m_{t}}\left(1-e^{-f_{t}}\right) w_{t} N_{t}
$$

To incorporate the effects of hypoxia, we introduce three potential parametric adjustments when a zone is hypoxic:

$$
\begin{aligned}
& \widetilde{m_{t}}=\left(1+\Delta_{m}\right) m_{t} \\
& \widetilde{q_{t}}=\left(1+\Delta_{q}\right) q \\
& \widetilde{\delta_{t}}=\left(1-\Delta_{\delta}\right) \delta .
\end{aligned}
$$

For catchability, it is important to note that when a fishing zone is hypoxic, the entire zone does not become uninhabitable by shrimp; rather, shrimp can aggregate along patchy edges 
separating hypoxic from non-hypoxic waters. We model hypoxia itself as having the potential to turn on in summer months and concentrate in the middle band of our nine-zone grid. We assume that the two zones on the offshore corners are never hypoxic; everything else can become hypoxic but with higher probability in the middle area. We use draws from a uniform distribution to assign actual hypoxia values in each simulation. Specifically, we assign "severe" hypoxia to the middle zone June through September and draw from $\mathrm{U}(0,4)$ for each month and round to the nearest whole number. If the rounded draw is 2 or greater in a month, the zone is considered to be hypoxic. For May and October, we draw from $\mathrm{U}(0,2)$ and do the same. For "moderate" hypoxia zones, we draw from $\mathrm{U}(0,2)$ for June through September and follow the same rule. Moderate zones are assumed non-hypoxic in May and October. Nonhypoxic zones are simply never hypoxic. Figure 1 depicts our grid and hypoxic severity graphically.

The economic model follows Smith, Sanchirico, and Wilen (2009) and Smith et al. (2010) in which individual fishermen are price-takers in a limited-entry fishery. Each day individuals $(i=1,2, \ldots, I)$ choose whether to fish and, if they fish, where to fish. Denoting fishing site as $j=1,2 \ldots, J$, we also model the alternative of not fishing $(j=0)$. We assume fishing sites and zones are equivalent such that $J=9$ for our simulations. The utility of each choice $(U)$ can be broken into a deterministic and random component:

$$
U_{i j t}=v_{i t j}+\eta_{i j t} \text {. }
$$

We assume that the $\eta_{i j t}$ 's are i.i.d. Type I Extreme Value, which provides a closed-form expression for probabilities of fishing in each location on each occasion. The deterministic portion of utility from not fishing is the value of some outside opportunity $(\alpha)$, which reflects the value of leisure or wages from another employment activity. This term can also accommodate what might be considered non-economic motives, such as attachment to the fishing lifestyle (Smith et al. 2010). The deterministic portion of a fishing alternative is the 
profitability of fishing, which includes revenues from fishing, a fixed cost of taking a trip (bait, ice, etc.) denoted by $c$, and travel cost $(\phi l)$ :

$$
v_{i t j}=\left\{\begin{array}{l}
\alpha, \text { for } j=0 \\
p_{t} h_{i j t}-c-\phi l_{i j}, \text { for } j=1,2,3, \ldots J
\end{array}\right.
$$

where $h$ is individual expected harvest, $p$ is price of fish, and $l_{i j}$ is the pairwise length of round-trip travel distance from individual $i$ 's port to patch $j$. These distances can introduce a source of spatial variation in the spatial gradient of costs that is potentially independent of spatial variation in the bio-physical gradient. For simplicity we assume that all fishermen start at the same port. ${ }^{2}$ Price is linear in fish size according to:

$$
p_{t}=\overline{p_{t}}+\varphi w_{t},
$$

where $\overline{p_{t}}$ is a base price and $\varphi$ is a per-pound premium for larger individuals. Although it is reasonable to assume that individual fishermen are price takers, in future work we plan to endogenize market price to further explore channels through which hypoxia can affect outcomes. We assume that expected individual harvest is proportional to stock in the zone scaled by catchability, which allows spatio-temporal fishing effort to respond to spatiotemporal variation in abundance. Outside opportunities $(\alpha)$ could also vary systematically over space and time in ways that are uncorrelated with spatial-dynamic processes in the water, but we assume this away initially. The trip fixed cost parameter, $c$, can also reflect non-market attachment to fishing as an occupation (Smith et al. 2010). Positive responsiveness of fishing effort to expected revenues is consistent with the discrete choice literature on fisheries in general, the particular findings of Huang et al. (2012) for North Carolina brown shrimp, and the model of spatial choices for the Gulf shrimp fleet in Ran, Keithly, and Kazmierczak (2011).

In the static random utility maximization framework (RUM) due to McFadden (1974), an individual is assumed to select the choice with the highest utility for each choice occasion. 
With a large number of individual fishermen making fishing choices, we assume away

idiosyncratic errors ${ }^{3}$ and use the logit probabilities to predict spatial effort in each period:

$$
E_{t, j}=I\left(\frac{e^{v_{i, t, j}}}{\sum_{k=0}^{J} e^{v_{i, t, k}}}\right)
$$

Equation (13) closes the model such that the spatial-dynamic biological system is fully coupled with the economic system, recognizing that hypoxia and prices are assumed exogenous. Baseline parameter values appear in the Appendix. The parameters follow Huang and Smith (2011) for the biological system and are based on meaningful parts of the parameter space in Smith et al. (2010) for the economic system. Nevertheless, the focus of the simulations is to produce qualitative, not quantitative, predictions.

\section{Findings from Numerical Experiments}

Because recruitment is exogenous and shrimp are an annual species, each year in a simulation is an experimental replicate. To understand what we might see in aggregate data across years, we run sets of 50-year simulations. This framework allows us to interpret output on an annual scale as 50 independent observations. Across observations within a 50-year simulation, all variation is due to stochastic recruitment and stochastic occurrence of hypoxia. We run five sets of 50-year simulations for each hypoxia effect: the mortality effect, the catchability effect, and the growth effect. Additionally, we run five sets of simulations that combine all three effects for a total of 20 50-year simulations.

Figure 2 illustrates some of the content of a single year of simulation using the combined effect of growth, catchability, and mortality. Additional snapshots of the mortality, growth, and catchability effects, depicted individually, are in the Appendix. There are several lessons in figure 2. First, the aggregate catch difference adding up across all zones varies dynamically and departs nonlinearly from the counterfactual catch. Second, the effects of hypoxia can even be non-monotonic over time at the aggregate level. That is, catch with hypoxia could be 
higher or lower than catch without hypoxia, depending on the time period. Third, the bioeconomic effects of hypoxia propagate to zones that are never hypoxic. Zones that do not experience hypoxia are influenced by the sorting that takes place in response to zones that do experience hypoxia. This raises questions about treatment-effects approaches and whether is it possible to construct a valid statistical counterfactual in any setting. Spatial-dynamic problems lead to general equilibrium effects that contaminate potential controls even in the absence of bio-physical dispersal, which we have assumed away in this application. Fourth, effort peaks at different times across zones, reflecting a sequencing of resources that comes about through myopic decision-making (by construction the agents are not forward-looking). What might appear to be chattering in the results comes out of real structural aspects of the model. Transitions from non-hypoxic to hypoxic conditions occur suddenly in the model (at the beginning of a month). Consequently, incentives, associated behavior, and resulting catch change non-marginally. These non-marginal changes then reverberate through the spatialdynamic system. In real observational data, these transitions are likely to be more gradual, but the drivers may be similar.

Natural scientists have struggled to understand the effects of hypoxia on fisheries using aggregate-level data. What is missing from these attempts is the ability to observe (or meaningfully control for) the counterfactual case of the fishery without hypoxia. With numerical experiments, we can observe the counterfactual by construction. We first analyze the effect of hypoxia on annual total landings. For the mortality, growth, and combined effect simulations, hypoxia always reduces total landings relative to the counterfactual. For the catchability effect, hypoxia always increases landings relative to the counterfactual.

Correlations of the severity of hypoxia with annual percent change in landings are consistent with individual simulation results (table 1). When hypoxia is more severe, percent loss is higher for mortality, growth, and combined effects. The opposite is true for the catchability 
effect. All correlations are statistically significant. An important caveat here is that the combined effect could be parameterized in such a way that the catchability effect ends up dominating the other two effects, so if all three hypoxia effects exist in nature, the net effect may still be ambiguous. Under some reasonable parameters, hypoxia could actually be good for the fishery.

Numerical experiments also allow us to build hypothetical data sets similar to those that are presented to researchers in the real world. Empirically, at the annual level we observe variation in the severity of hypoxia and total landings. Numerical experiments provide some evidence that the severity of hypoxia is correlated with total annual landings. When hypoxia has a mortality effect, a growth effect, or combines all three effects, total landings are negatively correlated with the degree of hypoxia at the annual level (table 2). These correlations are only significant in four cases, and there is one positive, though not significant, exception. The counterfactual non-hypoxic simulations help us to understand lack of significance. None of counterfactuals are significant at the $5 \%$ level, and only one is significant at the $10 \%$ level. This is as expected; hypoxia by construction does not affect the counterfactual. However, random variation in the counterfactuals leads to some positive and negative correlations. When they are negative, the corresponding hypoxia simulations are more likely to be significant; when they are positive (or near zero) the hypoxia effects are smaller in magnitude. These results suggest that aggregate-level data may not reveal hypoxic effects on the fishery even when these effects are substantial.

Compared to the mortality and growth effects, the catchability effect is more ambiguous. Increased catchability, ceteris paribus, increases landings. But it also means shrimp are caught sooner, when they are smaller. We know that this leads to increases in catch relative to the counterfactual from table 1 , but measured across years with data only on severity of hypoxia, 
the effect is not clearly detectable. Four out of five correlations are positive in the hypoxia case, but all are small in magnitude and none are significant.

The most robust finding across all simulations at the aggregate level is that annual average shrimp size and annual total landings are negatively correlated. In all 20 sets of simulations, the correlation is negative and statistically significant for both the hypoxic case and the counterfactual non-hypoxic case (40 correlation coefficients in total, ranging from -0.67 to 0.31). At first glance, this result is surprising. After all, larger shrimp weigh more, so one might expect larger catches to be comprised of larger individuals, on average. However, within-season dynamics lead to the opposite outcome. A more successful recruitment year translates into higher expected revenues because the shrimp stock is larger. These higher revenues, in turn, attract more fishing effort early in the season before the shrimp have grown as large as they otherwise would have. This pattern is consistent with growth overfishing and is similar to how poor regulations can incentivize landing suboptimal quality fish (Larkin and Sylvia 1999). The result is that the catch composition tilts toward smaller size classes. The simulations thus illustrate a classic example of growth overfishing. Reinforcing this effect is natural mortality; a fraction of individuals caught later in the season, though larger in size, would have died anyway. Because this result appears in both hypoxic and counterfactual nonhypoxic cases, it is not due to hypoxia but is a key mechanism that will influence our ability to understand hypoxia's effects on the fishery.

Comparing hypoxic cases to counterfactual cases, the mortality, growth, and combined effects of hypoxia decrease average shrimp size landed, while the catchability effect alone increases average shrimp size landed. This finding is important in light of growth overfishing. For the mortality effect, relative abundance in non-hypoxic zones is higher and attracts more fishing effort at an earlier stage of the year. While dampening of fishing mortality within hypoxic areas does allow surviving shrimp to grow to larger sizes, the effect is insufficient to 
compensate for the larger harvests of smaller shrimp in other areas. For the growth effect, impacts on non-hypoxic and hypoxic areas are reinforcing; higher relative abundance in nonhypoxic zones attracts more effort in earlier periods while shrimp within the hypoxic zone are growing more slowly and, conditional on time of harvest, are smaller. The catchability effect has the opposite impact on average size because it serves to ameliorate growth overfishing. In reality, the hypoxic area is patchy, so the catchability effect reflects concentrations of shrimp along edges of hypoxic patches within a fishing zone. Concentrating effort in zones that contain hypoxic waters in the short run allows shrimp to grow larger in other areas, although it is surprising that this effect is large enough to overcome growth overfishing within the hypoxic zones.

In contrast, looking only at the severity of hypoxia and shrimp size, the bioeconomic simulations produce results similar to the somewhat ambiguous case of total landings (table 3). Under growth, mortality, and combined effects hypoxic severity is negatively correlated with average size, but correlations are mostly not significant. Correlations are positive, but not significant, for the catchability effect. Moreover, as in Table 2, the correlations that are significant in Table 3 tend to be ones that coincidently are reinforced as shown in the counterfactual simulations. Thus, we do not have a strong expectation about the empirical relationship between average size at the aggregate level and severity of hypoxia.

Overall, the simulation results make sharp predictions about the effects of hypoxia on the fishery if one were to observe the counterfactual or had some means of constructing it. Moreover, the results strongly suggest that total landings and average shrimp size will be negatively correlated. However, in cases in which one cannot construct the counterfactual, inferences about the effect of hypoxia from aggregate data are likely to be confounded. In the next section, we explore some of the aggregate-level data and compare these data to the 
simulations to highlight some of the ways in which these various confounding factors manifest in the data.

\section{Empirical Application-The Gulf of Mexico Brown Shrimp Fishery}

Brown shrimp is one of three major shrimp fisheries in the Gulf of Mexico (the others being white and pink). Brown shrimp landings in the Gulf over the past decade ranged from 33,000 to 71,000 metric tons with landed value between $\$ 137$ million and $\$ 355$ million (Asche et al. 2012). Brown shrimp is a highly fecund annual species, so recruitment overfishing is not an issue. In fact, recruitment is considered to be exogenous, and the only overfishing concern is growth overfishing. Nevertheless, the brown shrimp fishery faces significant challenges. Beyond the potential negative effects of hypoxia, the Gulf shrimp fisheries have experienced other external shocks (e.g., Hurricane Katrina, the Deepwater Horizon incident), increased competition from imported (mostly farmed) shrimp (80\% of the U.S. market is imports despite the large domestic fishery), rising fuel costs (shrimp trawling is fuel-intensive), and environmental regulations that increase costs (Mukherjee and Segerson 2011; Asche et al. 2012). The fishery is regulated with limited entry, gear restrictions, and some seasonal closures. Most notable is the Texas seasonal closure (typically 45 days starting in late May), which attempts to allow shrimp to grow to larger, more valuable sizes.

We use landings data from NOAA's SHRCOM database that records, on a daily basis, volume landed and landed value for each shrimp species by each vessel within each of ten size classes. Two of these size classes are not useful for our analysis (unclassified and pieces), but the remaining classes correspond to number of shrimp per pound: 1) <15, 2) 15-20, 3) 20 $25,4) 25-30,5) 30-40,6) 40-50,7) 50-67,8)>67$. Using midpoints of each size class, one can covert to grams per individual for classes 2 through 7 (classes 1 and 8 require making an additional assumption of how large or small the mean shrimp is within the class). We use data 
on hypoxia from Craig (2012), who estimates the aerial extent of hypoxia in each year using NOAA's summer trawl surveys (SEAMAP).

The most striking feature of the aggregate data is that the severity of hypoxia is negatively correlated with total landings (figure 3). As noted above, this result occurs in some of the numerical experiments, but it is not robust across the different specifications. Whether this pattern reflects negative impacts of hypoxia on the fishery or broader trends is unclear. Hypoxia fluctuates inter-annually but generally has trended upward over time (figure 4). Note that 1989 is not a true zero but was a year in which no data were collected. During the sample period, fuel prices have also trended up, while real shrimp prices have trended down. So, it is difficult to know how to assess the annual-level negative correlation in figure 3.

Despite the negative correlation between catches and hypoxic severity, we do not see negative correlation between hypoxic severity and average shrimp size landed. In fact, we see positive correlations (linear-linear, log-linear, and log-log), though none are statistically significant. This aggregate outcome is somewhat consistent with our findings from the bioeconomic simulations. In simulations, hypoxia appears to have only weak impacts on average shrimp size due, in part, to feedbacks within the spatial-dynamic system. Moreover, the different hypoxic effects can have different signs (catchability being positive while growth and mortality being negative).

As we found in the spatial-dynamic numerical experiments, average shrimp size and total landings are negatively correlated at the annual level (figure 5). While this evidence is not causal, it is suggestive of the pattern of growth overfishing described above. In a good year, fishermen fish more intensively early in the season and catch a larger share of small shrimp. If more severe hypoxia is negatively correlated with a good year for the fishery, then isolating the hypoxia data from pure quantity data will be problematic. 
To explore potential hypoxic effects in more detail, we next examine shrimp size distributions. A key confounding factor is that hypoxic severity is negatively correlated with landings. This translates into decreases in landings across size classes. In percentage terms, the effects may be ambiguous. Consider two years with dramatically different levels of hypoxia: 2008 (severe) and 2009 (moderate). The percentage of biomass landed in the largest class is lower in the severe year, and the percentage of biomass landed in the smallest class is higher in the severe year (figure 6). However, the effects are the opposite in the second smallest and second largest size classes.

In levels, we begin to understand the difficulty in isolating the effects of hypoxia on the shrimp fishery. Severity of hypoxia is negatively correlated with landings in most of the size classes (table 4). Since landings are not distributed uniformly, it is unclear what a meaningful change in the size distribution of landings would be. A small change in large shrimp landings could matter more than a large change in small shrimp landings, or vice versa.

\section{Discussion}

The effects of hypoxia on fisheries are far from obvious. While the costs of controlling nutrient pollution are substantial and involve their own set of spatial-dynamic complications (Ribaudo et al. 2005; Gren, Savchuk, and Janson 2013), understanding hypoxic effects on fisheries is one part of estimating benefits of these reductions. Here we examine potential effects of hypoxia using a spatial-dynamic bioeconomic simulation and find strong indications that growth and mortality effects of hypoxia will lead to decreases in harvests. The catchability effect, on the other hand, would increase harvests. All of these effects are obscured in aggregate-level data that do not contain a clear indication of the counterfactual. Most aggregate-level predictions are only mildly suggestive of hypoxia’s effects. Empirical data from the Gulf of Mexico brown shrimp fishery appear consistent with these somewhat 
ambiguous predictions. The simulations and the empirical data are also consistent with a pattern of persistent growth overfishing.

For those, including ourselves, inclined to use a natural experiments (treatment effects) framework to isolate the effects of hypoxia on the Gulf shrimp fishery, the complexity of simulated outcomes is not welcome news. With treatment effects that vary over time and space and the potential contamination of control sites through re-sorting of the fishing fleet, there is ambiguity about shrimp quantities even when the ecological system is well observed. One alternative approach we are employing in ongoing work is to use time series analysis of prices. Here, theoretical predictions are much sharper because all of the biological effects of hypoxia predict a relative increase in small shrimp, which, in turn, predicts a decrease in relative prices of small-to-large shrimp. In that sense, the market ultimately may be a better judge of the severity of an ecological disturbance than an analyst's attempts to construct a counterfactual. A second alternative is to harness the structural information in our bioeconomic simulation model as identifying assumptions. It may be that biologists know enough about baseline parameters to use the simulations to predict outcomes and estimate structural hypoxia parameters in a framework similar to Smith, Zhang, and Coleman (2008). Whichever research approach is pursued, it appears that we are far from having an empirical foundation for benefit-cost analysis of nutrient reductions in the Mississippi Basin. 


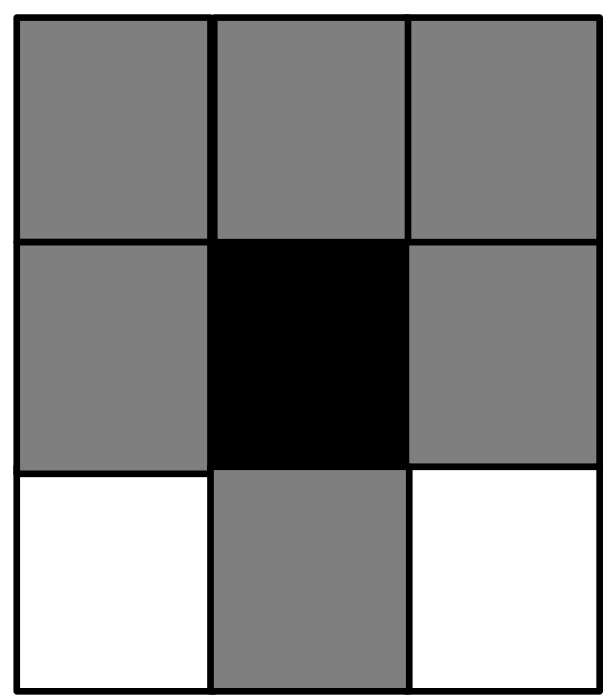

Figure 1. Average Hypoxic Severity by Zone

Note: White is non-hypoxic, grey is moderate, black is severe. 

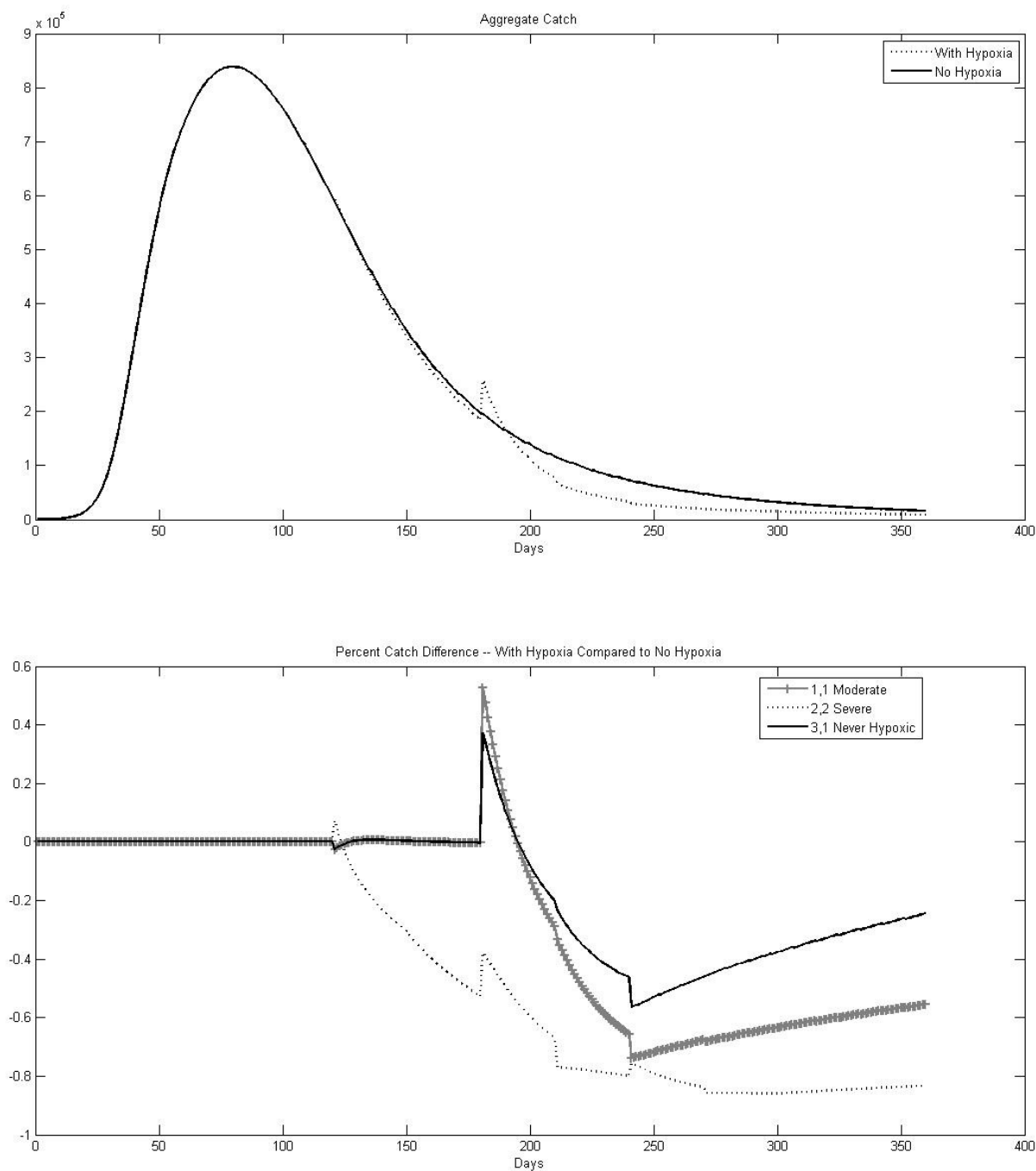

Figure 2. A Single-year Snapshot of the Combined Effect

(Mortality, Catchability, and Growth) in a Bioeconomic Simulation

Note: The top panel depicts total catch (with hypoxia) and total counterfactual catch (no hypoxia) throughout the year. The bottom panel depicts the instantaneous percent change in catch using no hypoxia as the baseline in three of the nine zones that experience hypoxia moderately, severely, and never. 


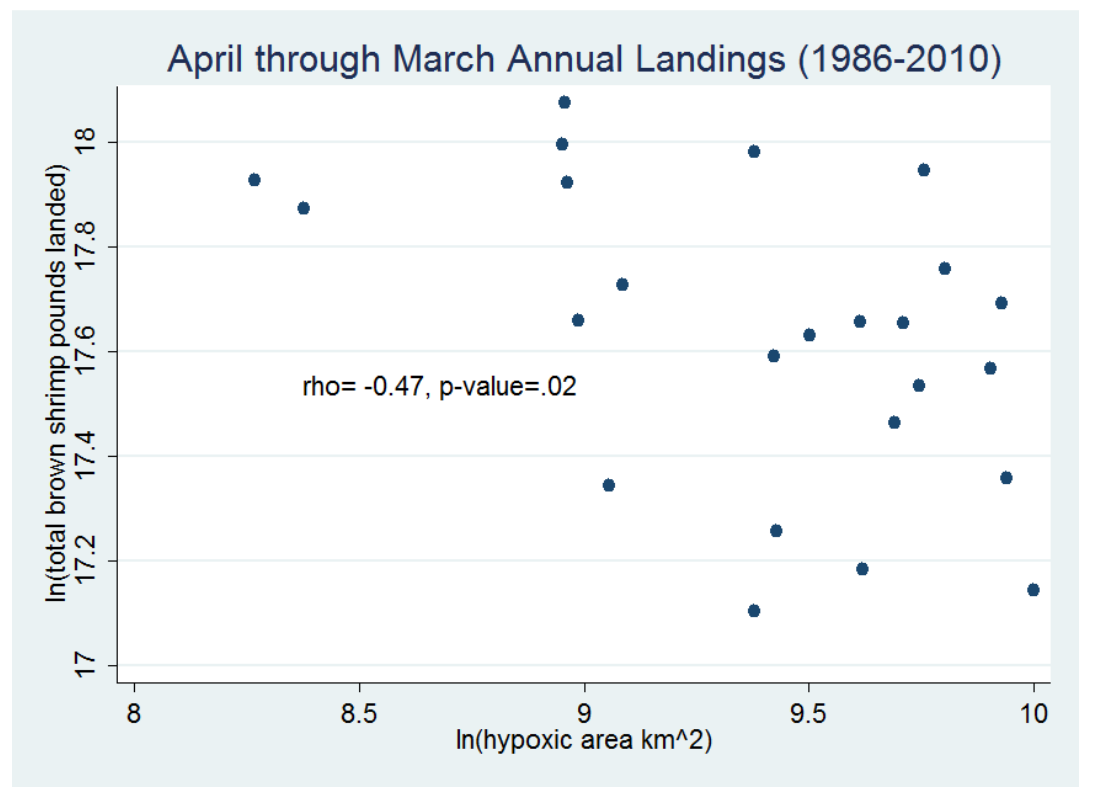

Figure 3. Gulf of Mexico Brown Shrimp Annual Landings and Hypoxia

Note: Excludes landings for which size class is not reported and the category "pieces." 


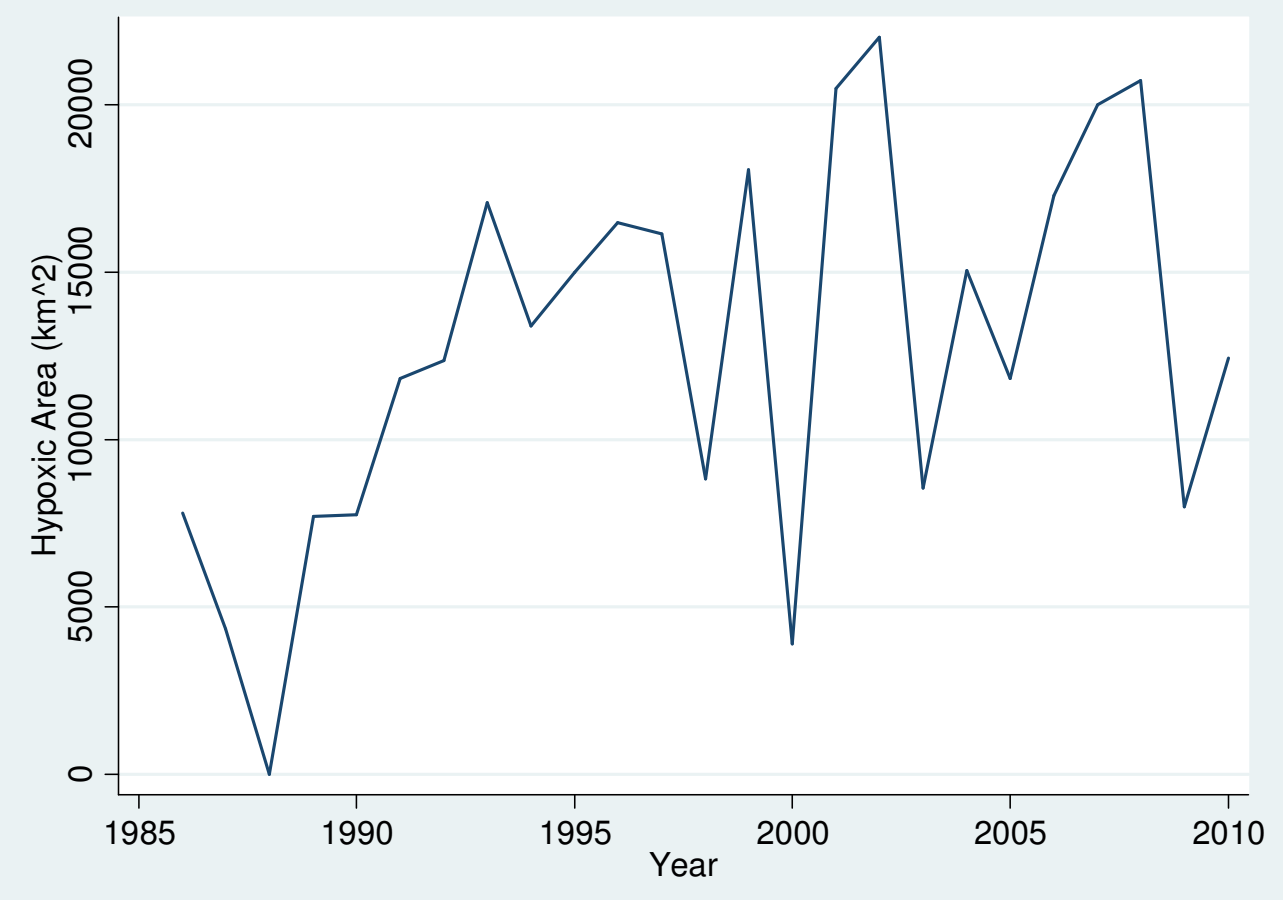

Figure 4. Hypoxic Area Over Time 


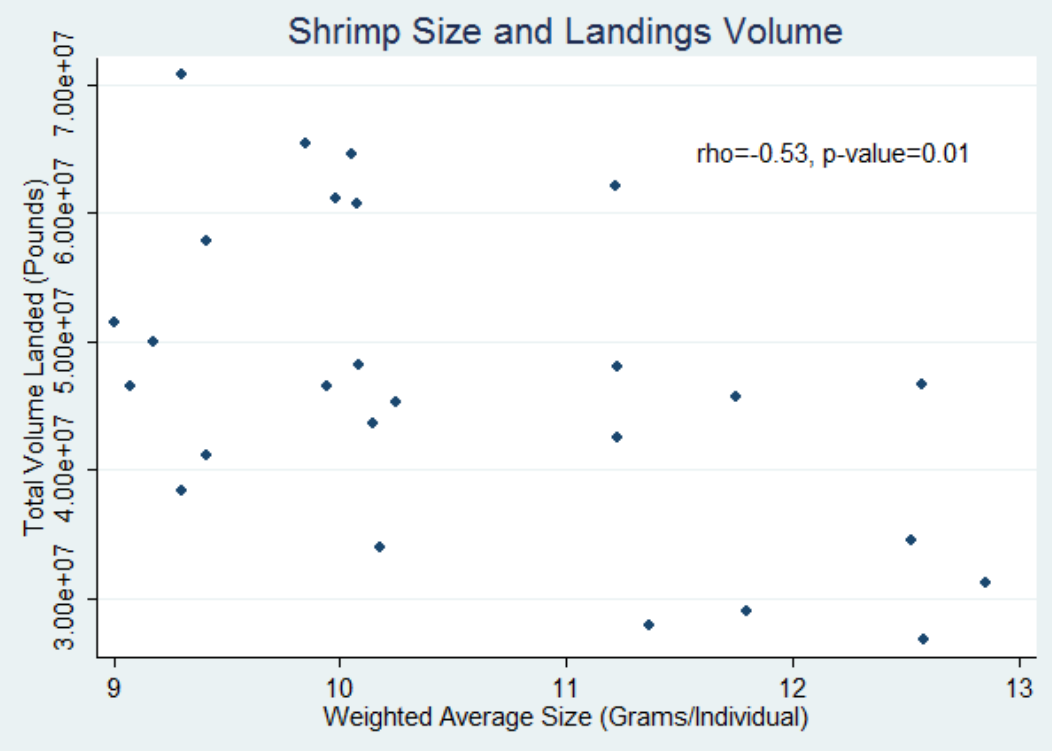

Figure 5. Shrimp Size and Landings are Negatively Correlated

Note: Excludes landings for which size class is not reported and the category "pieces." 

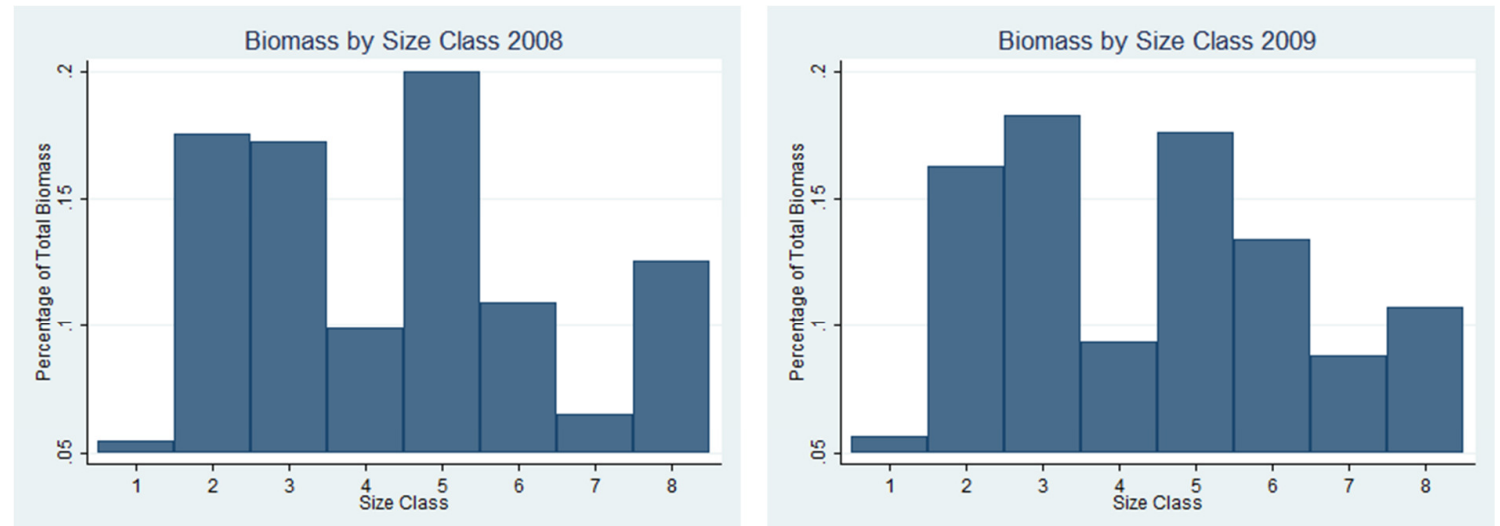

Figure 6. Percentage Landings in Severe (2008) and Moderate (2009) Hypoxia Years 


\section{Table 1}

Correlations of Simulated Percent Change in Annual Total Landings Due to Hypoxia with Severity of Hypoxia

\begin{tabular}{|c|c|c|c|c|c|c|c|c|}
\hline Sim \# & Mortality & & Catchabil & & Growth & & Combine & \\
\hline 1 & 0.82 & $* *$ & -0.57 & $* *$ & 0.58 & $* *$ & 0.66 & $* *$ \\
\hline 2 & 0.77 & $* *$ & -0.61 & $* *$ & 0.62 & $* *$ & 0.74 & $* *$ \\
\hline 3 & 0.78 & $* *$ & -0.67 & $* *$ & 0.60 & $* *$ & 0.72 & $* *$ \\
\hline 4 & 0.83 & $* *$ & -0.66 & $* *$ & 0.62 & $* *$ & 0.75 & $* *$ \\
\hline 5 & 0.82 & $* *$ & -0.69 & $* *$ & 0.66 & $* *$ & 0.69 & $* *$ \\
\hline
\end{tabular}


Table 2

Correlations of Simulated Annual Total Landings with Severity of Hypoxia

\begin{tabular}{|c|c|c|c|c|c|c|}
\hline \multirow[b]{2}{*}{ Sim \# } & \multirow{2}{*}{\multicolumn{2}{|c|}{ Mortality }} & - & & \multirow{2}{*}{\multicolumn{2}{|c|}{ Combined }} \\
\hline & & & Catchability & Growth & & \\
\hline \multicolumn{7}{|l|}{ Hypoxic } \\
\hline 1 & -0.35 & $* *$ & 0.01 & -0.14 & -0.31 & $* *$ \\
\hline 2 & -0.08 & & 0.01 & -0.12 & -0.10 & \\
\hline 3 & -0.14 & & 0.17 & -0.01 & -0.34 & $* *$ \\
\hline 4 & -0.35 & $* *$ & -0.16 & -0.23 & -0.10 & \\
\hline 5 & -0.09 & & 0.16 & -0.05 & 0.03 & \\
\hline \multicolumn{7}{|c|}{ Counterfactual } \\
\hline 1 & -0.25 & $*$ & -0.05 & -0.05 & -0.17 & \\
\hline 2 & 0.03 & & -0.05 & -0.02 & 0.07 & \\
\hline 3 & -0.04 & & 0.11 & 0.08 & -0.17 & \\
\hline 4 & -0.21 & & -0.21 & -0.11 & 0.04 & \\
\hline 5 & 0.03 & & 0.10 & 0.04 & 0.22 & \\
\hline
\end{tabular}

** and * indicate significance at the $5 \%$ and $10 \%$ level, respectively. 
Table 3

Correlations of Simulated Annual Average Shrimp Size with Hypoxic Severity

\begin{tabular}{|c|c|c|c|c|c|c|}
\hline Sim \# & Mortality & & Catchability & Growth & Combinec & \\
\hline \multicolumn{7}{|l|}{ Hypoxic } \\
\hline 1 & 0.02 & & 0.02 & -0.16 & -0.21 & \\
\hline 2 & -0.36 & $* *$ & 0.19 & -0.15 & 0.06 & \\
\hline 3 & -0.05 & & 0.05 & -0.16 & -0.13 & \\
\hline 4 & -0.01 & & 0.14 & 0.08 & -0.20 & \\
\hline 5 & -0.15 & & 0.05 & -0.23 & -0.32 & $* *$ \\
\hline \multicolumn{7}{|c|}{ Counterfactual } \\
\hline 1 & 0.12 & & -0.01 & -0.05 & -0.07 & \\
\hline 2 & -0.24 & $*$ & 0.16 & -0.04 & 0.26 & \\
\hline 3 & 0.05 & & 0.01 & -0.05 & 0.07 & \\
\hline 4 & 0.10 & & 0.11 & 0.18 & -0.04 & \\
\hline 5 & -0.04 & & 0.01 & -0.09 & -0.15 & \\
\hline
\end{tabular}


Table 4

Correlations of Severity of Hypoxia with Total Landings by Size

\begin{tabular}{|c|c|c|}
\hline Pairwise Correlatic & $\begin{array}{c}\text { oxic ar } \\
\text { Rho }\end{array}$ & $\begin{array}{l}\text { with: } \\
\text { P-Value }\end{array}$ \\
\hline In(total landings) & -0.47 & 0.02 \\
\hline In(landings size1) & 0.09 & 0.67 \\
\hline In(landings size2) & -0.32 & 0.13 \\
\hline In(landings size3) & -0.29 & 0.17 \\
\hline In(landings size4) & -0.46 & 0.02 \\
\hline In(landings size5) & -0.52 & 0.01 \\
\hline In(landings size6) & -0.44 & 0.03 \\
\hline In(landings size7) & -0.46 & 0.03 \\
\hline In(landings size8) & -0.31 & 0.14 \\
\hline
\end{tabular}




\section{Appendix}
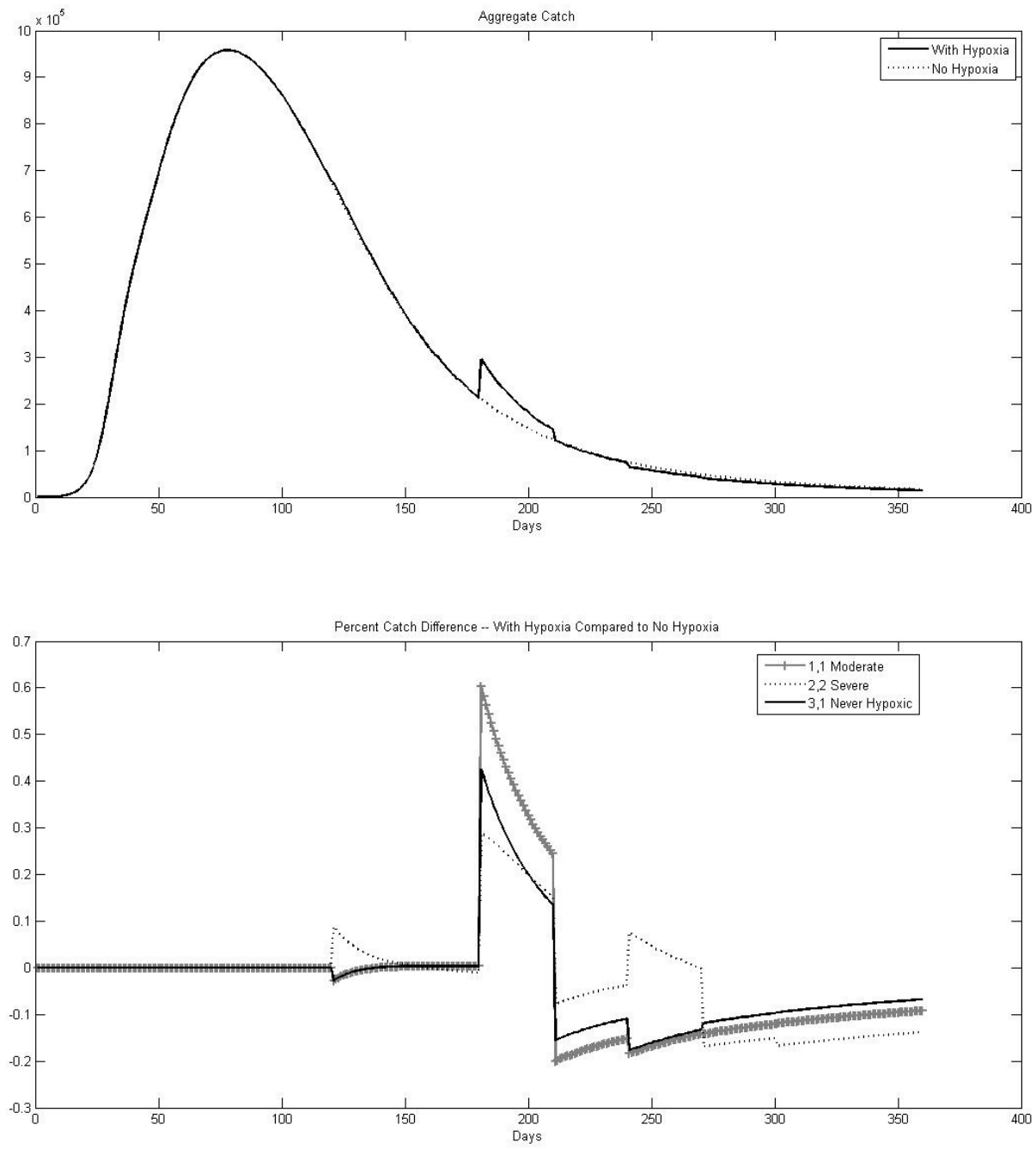

Figure A1. A Single-year Snapshot of the Catchability Effect in a Bioeconomic Simulation

Note: The top panel depicts total catch (with hypoxia) and total counterfactual catch (no hypoxia) throughout the year. The bottom panel depicts the instantaneous percent change in catch using no hypoxia as the baseline in three of the nine zones that experience hypoxia moderately, severely, and never. 

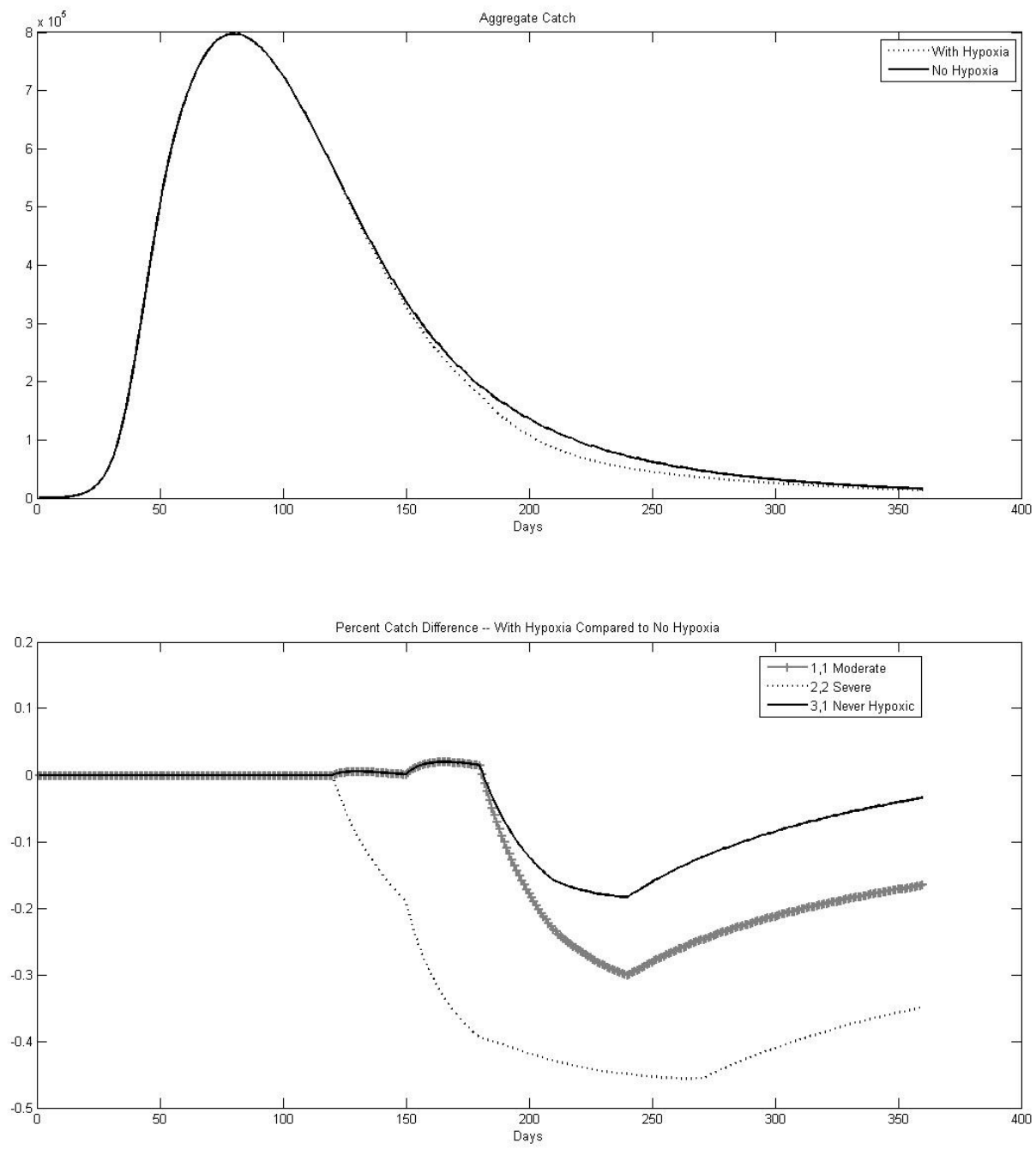

Figure A2. A Single-year Snapshot of the Growth Effect in a Bioeconomic Simulation

Note: The top panel depicts total catch (with hypoxia) and total counterfactual catch (no hypoxia) throughout the year. The bottom panel depicts the instantaneous percent change in catch using no hypoxia as the baseline in three of the nine zones that experience hypoxia moderately, severely, and never. 

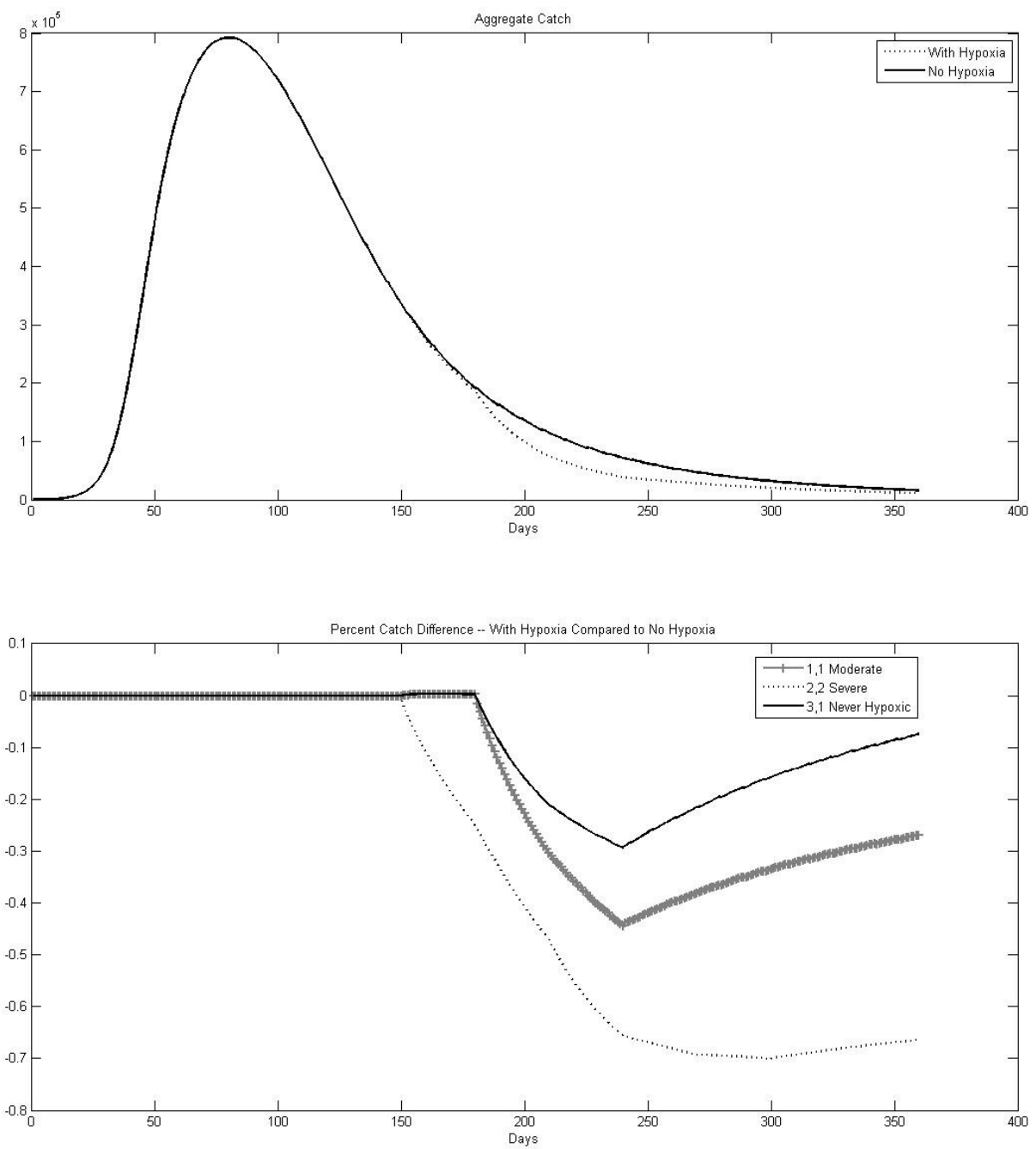

Figure A3. A Single-year Snapshot of the Mortality Effect in a Bioeconomic Simulation

Note: The top panel depicts total catch (with hypoxia) and total counterfactual catch (no hypoxia) throughout the year. The bottom panel depicts the instantaneous percent change in catch using no hypoxia as the baseline in three of the nine zones that experience hypoxia moderately, severely, and never. 


\section{Table A1}

Baseline Parameter Values in the Simulation Model

\section{Simulation Structure}

J

n

T

Y

\section{Spatial Structure}

$\theta$

I

Hypoxia

\section{Randomness}

$\varepsilon_{j, y}$

$\eta_{i j t}$

Hypoxia Moderate

Hypoxia Severe
9 Number of locations

1,500 Number of fishing vessels

360 Days within each cohort simulation

50 Years for a complete simulation replicate

$\begin{array}{llll}0.3 & 0.3 & 0.3 & \text { Recruit allocation } \\ 0.4 & 0.4 & 0.4 & \\ 0.3 & 0.3 & 0.3 & \end{array}$

$\begin{array}{llll}1.41 & 2.24 & 3.16 & \text { Distances }\end{array}$

$\begin{array}{lll}1.00 & 2.00 \quad 3.00\end{array}$

$1.41 \quad 2.24 \quad 3.16$

$\begin{array}{lll}\text { Moderate } & \text { Moderate } & \text { Moderate } \\ \text { Moderate } & \text { Severe } & \text { Moderate } \\ \text { None } & \text { Moderate } & \text { None }\end{array}$

\section{Biology}

$L_{\infty}$

$\delta$

$\beta$

$\mu$

$\omega$

$\gamma$

$N_{0}$

\section{Economics}

$q$

$\alpha$

$c$

$\bar{\phi}$

$\varphi$ $\sim$ Uniform $(0,1)$

$\sim$ Type I Extreme Value $(0,1)$

$\sim$ Uniform $(0,2)$

$\sim$ Uniform $(0,4)$
Stochastic recruitment

Profit shocks in sorting vessels

177.7 von-Bertallanfy terminal length

0.104 von-Bertallanfy growth

1.4866 Base natural mortality

-1.1163 Time decay natural mortality

0.000819 Scaling allometric parameter

2.94 Exponential allometric parameter

20,000,000 Deterministic initial recruits

\footnotetext{
0.0005 Catchability

1 Fishing outside opportunity cost

0 Fishing quasi-fixed trip cost (not seperately identified from $\alpha$ )

3 Fishing travel cost

1 Baseline price

0.1 Slope of size-based pricing curve
} 
Hypoxia

$\Delta_{m}$

75\% Mortality adjustment

$\Delta_{q}$

$\Delta_{\delta}$

15\% Catchability adjustment

0.0025 Growth adjustment 


\section{References}

Andam, K. S., P. J. Ferraro, A. Pfaff, G. A. Sanchez-Azofeifa, and J.Al. Robalino. 2008. "Measuring the Effectiveness of Protected Area Networks in Reducing Deforestation." Proceedings of the National Academy of Sciences 105:16089-94.

Asche, F., L.S. Bennear, A. Oglend, and M.D. Smith. 2012. “U.S. Shrimp Market Integration." Marine Resource Economics 27:181-92.

Auffhammer, M., A. M. Bento, and S. E. Lowe. 2009. "Measuring the Effects of the Clean Air Act Amendments on Ambient PM10 Concentrations: The Critical Importance of a Spatially Disaggregated Analysis." Journal of Environmental Economics and Management $58: 15-26$.

Caillouet Jr, C. W., R. A. Hart, and J.M. Nance, J. M. 2008. "Growth Overfishing in the Brown Shrimp Fishery of Texas, Louisiana, and Adjoining Gulf of Mexico EEZ.” Fisheries Research 92: 289-302.

Craig J.K. 2012. "Aggregation on the Edge: Effects of Hypoxia Avoidance on the Spatial Distribution of Brown Shrimp and Demersal Fishes in the Northern Gulf of Mexico." Marine Ecology Progress Series 445:75-95.

Craig J.K. and L.B. Crowder. 2005. "Hypoxia-induced Habitat Shifts and Energetic Consequences in Atlantic Croaker and Brown Shrimp on the Gulf of Mexico Shelf." Marine Ecology Progress Series 294: 79-94. 
Deng, X., J. Huang, E.Uchida, S. Rozelle, and J. Gibson. 2011. "Pressure Cookers or Pressure Valves: Do Roads Lead to Deforestation in China?" Journal of Environmental Economics and Management 61: 79-94.

Diaz, R.J. and R. Rosenberg. 2008. "Spreading Dead Zones and Consequences for Marine Ecosystems." Science 321: 926-29.

Gren I.-M., O.P. Savchuk, and T. Janson. 2013. "Cost-Effective Spatial and Dynamic Management of a Eutrophied Baltic Sea.” Marine Resource Economics 28: 263-84.

Huang, L., L.A.B. Nichols, J.K. Craig and M.D. Smith 2012. "Measuring Welfare Losses from Hypoxia: The Case of North Carolina Brown Shrimp.” Marine Resource Economics $27: 3-23$.

Huang L. and M.D. Smith. 2011. "Management of an Annual Fishery in the Presence of Ecological Stress: The Case of Shrimp and Hypoxia.” Ecological Economics 70: 688-97.

Huang L., M.D. Smith, and J.K. Craig. 2010. "Quantifying the Economic Effects of Hypoxia on a Fishery for Brown Shrimp Farfantepenaeus aztecus." Marine and Coastal Fisheries: Dynamics, Management, and Ecosystem Science 2: 232-48.

Knowler D, E.B. Barbier, and I.E. Strand. 2001. “An Open-access Model of Fisheries and Nutrient Enrichment in the Black Sea." Marine Resource Economics 16: 195-217. 
Larkin, S. and G. Sylvia. 1999. "Intrinsic Fish Characteristics and Production Efficiency." American Journal of Agricultural Economics 81:29-43.

Massey D.M., S.C. Newbold, and B. Gentner. 2006. "Valuing Water Quality Changes Using a Bioeconomic Model of a Coastal Recreational Fishery." Journal of Environmental Economics and Management 52: 482-500.

McFadden, D. ed. 1974. Conditional Logit Analysis of Qualitative Choice Behavior. New York: Academic Press.

Minello, T. J., R. J. Zimmerman, and E.X. Martinez. 1989. "Mortality of Young Brown Shrimp Penaeus aztecus in Estuarine Nurseries." Transactions of the American Fisheries Society 118: 693-708.

Mukherjee, Z. and K. Segerson. 2011. "Turtle Excluder Device Regulation and Shrimp Harvest: The Role of Behavioral and Market Responses." Marine Resource Economics 26:173-89.

Rabalais, N.N., R.E. Turner, and W.J. Wiseman, Jr. 2002. "Gulf of Mexico Hypoxia, a.k.a. ‘The Dead Zone.” Annual Review of Ecology and Systematics 33:235-63.

Ran, T., W. R. Keithly, and R.F. Kazmierczak. 2011. "Location Choice Behavior of Gulf of Mexico Shrimpers under Dynamic Economic Conditions." Journal of Agricultural and Applied Economics 43: 29-42. 
Ribaudo, M.O., R. Heimlich, and M. Peters. 2005. "Nitrogen Sources and Gulf Hypoxia: Potential for Environmental Credit Trading.” Ecological Economics 52:159-168.

Smith MD. 2007. "Generating Value in Habitat-dependent Fisheries: The Importance of Fishery Management Institutions.” Land Economics 83:59-73.

Smith, M.D., J. Lynham, J.N. Sanchirico, and J.A. Wilson. 2010. "Political Economy of Marine Reserves: Understanding the Role of Opportunity Costs." Proceedings of the National Academy of Sciences of the United States of America 107:18300-05.

Smith, M.D., J.N. Sanchirico, and J.E. Wilen. 2009. "The Economics of Spatial-dynamic Processes: Applications to Renewable Resources." Journal of Environmental Economics and Management 57:104-21.

Smith, M. D., J. Zhang, and F.C. Coleman. 2006. "Effectiveness of Marine Reserves for Large-scale Fisheries Management." Canadian Journal of Fisheries and Aquatic Sciences 63:153-164.

Smith, M.D., J. Zhang, and F.C. Coleman. 2008. "Econometric Modeling of Fisheries with Complex Life Histories: Avoiding Biological Management Failures.” Journal of Environmental Economics and Management 55:265-80.

Zimmerman, R.J. and J.M. Nance. 2001. "Effects of Hypoxia on the Shrimp Fishery of Louisiana and Texas." Coastal and Estuarine Studies 58:293-310. 
${ }^{1}$ Formally, we are assuming recruitment that is exogenous with respect to fishing pressure and hypoxia in the Gulf. This is a reasonable starting place because shrimp are highly fecund and recruitment appears to be independent of fishing pressure. To the extent that recruitment is regulated by mortality on young shrimp (Minello, Zimmerman, and Martinez 1989), low oxygen in the estuaries before shrimp reach the Gulf is a driver of recruitment variability. However, this phenomenon would still make recruitment exogenous from the perspective of what we model. It could also be that some late-recruiting young shrimp are exposed to hypoxia in the Gulf itself as they migrate offshore, which, in turn, would influence overall annual recruitment. This latter possibility would be problematic to infer from observational data.

${ }^{2}$ This assumption makes the simulations more tractable and easy to interpret. Especially for large offshore vessels in the Gulf, it may be that travel cost is not particularly important relative to the cost of being at sea and trawling. Model experiments with lower travel costs reach similar qualitative conclusions.

${ }^{3}$ The systematic portion of utility presumes optimizing behavior (profit maximization or some combination of profits and taste for the fishing lifestyle). The extent to which behavior diverges from optimization entirely could be captured in the random component as in Smith et al. (2010); a higher variance of the error means more random behavior and less optimizing behavior. Exploring this issue in the Gulf shrimp fisheries is beyond our scope. 